\title{
Growth and reproductive behaviour of chickpea (Cicer arietinum L.) as influenced by supplemental application of nitrogen, irrigation and hormone
}

\author{
Indrajit Roy ${ }^{1}$, Parimal Kanti Biswas ${ }^{1}$, Md. Hazrat Ali ${ }^{1}$, Md. Nazmul Haque ${ }^{1^{*}}$, Khursheda \\ Parvin $^{2}$ \\ ${ }^{1}$ Department of Agronomy, Faculty of Agriculture, Sher-e-Bangla Agricultural University, Sher-e-Bangla Nagar, Dhaka-1207, \\ Bangladesh \\ ${ }^{2}$ Department of Horticulture, Faculty of Agriculture, Sher-e-Bangla Agricultural University, Sher-e-Bangla Nagar, Dhaka-1207, \\ Bangladesh
}

\section{Article history \\ Received: 29 November 2015 \\ Accepted: 1 January 2016 \\ Published online: 18 January 2016}

(C) Roy et al. 2016

Editor

Bhupinder Dhir

Special Section

Soil amendments and crop productivity

Publisher

Horizon e-Publishing Group

Corresponding Author

Md. Nazmul Haque

$\bigotimes_{\text {sumon2539@gmail.com }}$

\begin{abstract}
The experiment was conducted during the period from 11 December, 2012 to 30 March 2013 to study the effect of supplementary nitrogen, irrigation and hormones on flower droppings, growth and reproductive behaviour of chickpea. Statistically significant variation was recorded for different growth and reproductive parameters. Results showed that BARI chola 9 gave the highest plant height, number of branches plant ${ }^{-1}$, dry matter content plant ${ }^{-1}$, pod remaining, pod length, seed yield and the lowest flower dropping, pod dropping, total dropping. Among the supplemental treatments, the highest plant height, number of branches plant $^{-1}$, dry matter content plant ${ }^{-1}$, pod remaining, pod length, seed yield and the lowest flower dropping, pod dropping, total dropping were found from supplemental irrigation + aqueous $\mathrm{N}$ before flowering. In case of treatment combination, the highest plant height $(39.31 \mathrm{~cm})$, number of branches plant ${ }^{-1}$ (6.53), dry matter content plant ${ }^{-1}$ (7.02 g), pod remaining (39.67 \%), pod length $(1.96 \mathrm{~cm})$, seed yield $\left(1.94 \mathrm{t} \mathrm{ha}^{-1}\right)$ and the lowest flower dropping (57.27 \%), pod dropping $(3.07 \%)$, total dropping $(60.33 \%)$ was recorded from BARI chola 9 with supplemental irrigation + aqueous $\mathrm{N}$ before flowering.
\end{abstract}

Keywords

Cicer arietinum L.; hormone; irrigation; nitrogen; seed yield

Roy, I., Biswas, P. K., Ali, M. H., Haque, M. N., and Parvin, K. 2016. Growth and reproductive behaviour of chickpea (Cicer arietinum L.) as influenced by supplemental application of nitrogen, irrigation and hormone. Plant Science Today 3(1): 30-40. http://dx.doi.org/10.14719/pst.2016.3.1.176

\section{Introduction}

Chickpea (Cicer arietinum L.), commonly known as gram, is one of the important pulse crops in Bangladesh. Today, chickpea is the $3^{\text {rd }}$ most important pulse crop and about 15\% of the world's total pulse productions belong to this crop (FAO, 2010). Among the major pulses that grown in
Bangladesh chickpea ranked $5^{\text {th }}$ in area and production but $2^{\text {nd }}$ in consumption priority (BBS, 2010).

Various environmental, physiological and pathological reasons are responsible for low yield of chickpea. Flower and pod droppings play an important role for the lower yield of chickpea. 
Aziz et al. (1960) reported 20-50\% flower and pod dropping in chickpea. Being leguminous in nature, chickpea needs low but optimum nitrogen during onset of flowering and podding. Mansoor (2007) noted that lack of attention on fertilizer application in proper way with appropriate amount is identified for lowering chickpea yields. Experimental findings revealed that pulse crop stop to nourish Rhizobia rather translocally energy towards development of flowers and pods. Thus, nitrogen fixation is totally ceased during reproductive stage which eventually hampers the development of reproductive traits. In this situation nitrogen given as basal to the crop is not sufficiently available to the plant for nourishing its flowers and pods thus seed yield value is lower (Patel et al., 1984; BARC, 2005). Triggering nitrogen at the plant demand would be attempt towards yield improvements of pulse (Deolankar, 2005; Mukesh, 2006).

Water deficiency has adverse effects on growth, average yield and crude protein in legume crops. The flowering stage is the most vulnerable stage for water stress and chickpea is somewhat tolerant to deficit water but susceptible to excess water (Miah et al., 1991). Adequate supply of irrigation water along with chemical fertilizer is essential for normal growth and yield of a crop (Ayallew and Tabbada, 1987; Kumar et al., 1995). On the other hand, chickpea is grown in rabi season when lack of water becomes a serious restriction specially after flowerings. Saraf et al. (1990) stated that excess and deficient moisture conditions both are detrimental and reduce yield of chickpea. Nayyar et al. (2006) reported that the flowering and pod setting stages appear to be the most sensitive stages to water stress. Supplemental irrigation was applied to compare the treatment with control for identification of its role in flower droppings.

Plant growth regulators (PGR's) are organic compounds, which in small amounts, somehow modify a given physiological plant process. It plays an essential role in many aspects of plant growth and development (Patil et al., 1987; Dharmender et al., 1996). PRH is an organic product of Natural Bio Agro Tech Co. (Pvt.) Ltd. (NBAT) - a JapanBangladesh Joint Venture agro based company. The product is a hormone made from fruits vinegar and other natural ingredients. The composition of the product is their patent. As per NBAI, the PRH is used in cereal crops, vegetables, fruit crops, cash crops, pulses and oil crops, fisheries, poultry and livestock (www.nbatbd.com). Studies showed that nitrogen and hormone have role to check droppings and hence application of nitrogen and PRH before flowering was included in the study. The main objective of the experiment was to check the reproductive behaviour specially flower droppings.

\section{Materials and Methods \\ Experimental site and climate}

A field experiment was conducted at Sher-e-Bangla Agricultural University, Dhaka, Bangladesh situated at $23^{\circ} 74^{\circ} \mathrm{N}$ latitude and $90^{\circ} 35^{\circ} \mathrm{E}$ longitude at an altitude of 8.6 meter above the sea level during the period from December 11, 2012 to March 30, 2013. The soil of the experimental site was sandy loam with $\mathrm{pH}$ and Cation Exchange capacity (CEC) 5.6 and $2.64 \mathrm{meq} / 100 \mathrm{~g}$ soil, respectively. The experimental site is under subtropical humid climatic conditions. The mean relative humidity, minimum temperature, maximum temperature and total rainfall during the crop growing period have been presented in Table 1.

\section{Experimental treatments}

The experiment was consists of two chickpea varieties viz., $\mathrm{V}_{1}$-BARI chola $8, \mathrm{~V}_{2}$-BARI chola 9 and five supplementary treatments i.e. $\mathrm{T}_{1^{-}}$(no spray at flowering and afterwards) (control), $\mathrm{T}_{2^{-}}$ supplemental irrigation before flowering, $\mathrm{T}_{3^{-}}$ supplemental irrigation + aqueous $\mathrm{N}$ before flowering, $\mathrm{T}_{4}$-PRH (a phytohormone) spray before flowering and $\mathrm{T}_{5}$-Kinetine spray before flowering.

\section{Planting materials, design and plot size}

The variety BARI chola 8 and BARI chola 9 were used as the test crops. The two factors experiment was laid out in split-plot design with three replications. The two varieties were assigned in the main plot and five supplementary treatments in sub-plot. The size of the each unit plot was $4.0 \mathrm{~m}$ $\times 3.2 \mathrm{~m}$. The space between two blocks and two plots were $1.0 \mathrm{~m}$ and $0.5 \mathrm{~m}$, respectively.

Table 1. Records of meteorological observation (monthly) for the period of experiment (December 2012-

March 2013)

\begin{tabular}{|c|c|c|c|c|c|}
\hline \multirow[t]{2}{*}{ Month } & \multirow{2}{*}{$\begin{array}{l}\text { Mean relative } \\
\text { humidity }(\%)\end{array}$} & \multicolumn{2}{|c|}{ Mean temperature ( $\left.{ }^{\circ} \mathrm{C}\right)$} & \multirow{2}{*}{$\begin{array}{l}\text { Total } \\
(\mathrm{mm})\end{array}$} & \multirow[t]{2}{*}{ rainfall } \\
\hline & & Minimum & Maximum & & \\
\hline December 2012 & 78.58 & 14.54 & 23.93 & 5 & \\
\hline January 2013 & 65.39 & 12.09 & 24.55 & 14 & \\
\hline February 2013 & 47.16 & 16.5 & 27.86 & 34 & \\
\hline March 2013 & 43.8 & 23.3 & 31.6 & 43.4 & \\
\hline
\end{tabular}




\section{Fertilizer application}

Urea, Triple super phosphate (TSP), Muriate of potash (MoP), gypsum, zinc sulphate and boric acid were used as a source of N, P, K, Ca, S and B, respectively. Urea, Triple super phosphate (TSP), Muriate of potash (MoP), gypsum, zinc sulphate and boric acid were applied at the rate of 50, 90, 40, 110, 7 and $10 \mathrm{~kg} \mathrm{ha}^{-1}$, respectively. All of the fertilizers except urea were applied during final land preparation.

\section{Supplemental irrigation before flowering (SIBF)}

Supplementary irrigation was applied before flowering and it was done at 15 February at 65 Days after Sowing (DAS). Selected 6 plots were provided with flood irrigation.

\section{SIBF + Aqueous $\mathrm{N}$ before flowering}

SIBF + Aqueous $\mathrm{N}$ before flowering was applied before flowering and done at 05 February at 55 DAS. Selected 6 plots were provided with flood irrigation and aqueous N. For aqueous nitrogen 153.6 g urea were mixed with 6 liter of water and sprayed in the plots.

\section{PRH (a phytohormone) spray before flowering}

PRH were sprayed before flowering and solution were made by adding 12 spoons of PRH with 6 liter of water and applied at 05 February at 55 DAS.

\section{Kinetine spray before flowering}

Kinetine (kinetine puriss CHR : 6Furfurylaminopurine, $\mathrm{C}_{10} \mathrm{H}_{5} \mathrm{OH}$ ) were sprayed before flowering and soluation were made by adding $150 \mathrm{mg}$ kinetene and $10 \mathrm{ml}$ ethanol $\left(\mathrm{C}_{6} \mathrm{H}_{5} \mathrm{OH}\right)$ with 6 liter of water and applied at 05 February at 55 DAS.

\section{Dry matter content plant ${ }^{-1}$}

After taking fresh weight at 60, 75, 90 and 105 DAS, the sample was sliced into very thin pieces and put into envelop then placed in oven maintained at $70^{\circ} \mathrm{C}$ for 72 hours. It was then transferred into desiccators and allowed to cool down at room temperature. The final dry matter content was taken by following formula:

$$
\begin{gathered}
\text { Dry matter } \\
\text { content }=
\end{gathered} \quad \frac{\text { Dry weight of plants }(\mathrm{g})}{\text { Fresh weight of plants }(\mathrm{g})} \times 100
$$

\section{Flower and pod dropping}

Flower and pod dropping was counted for 5 selected plants and recorded in each plot. Dropping of flower and pod was counted in every morning by using clean paper during flowering time and pod development stage, respectively and recorded.

\section{Statistical analysis}

Collected data were statistically analyzed to find out the level of significance using MSTAT-C computer package programme developed by Freed (1986). The mean differences were assessed by least significant difference (LSD) at 5\% level of probability (Gomez and Gomez, 1984).

\section{Results and Discussion \\ Plant height}

Varieties had significant effect on plant height of chickpea at different DAS (Figure 1). Plant height increased with advancing growing period irrespective of varieties. Plant height increased rapidly at the early stages of growth; however, rate of progression in height was slow at the later stages. Different varieties produced different plant height on the basis of their varietal characters and improved varieties is the first and foremost requirement for initiation and accelerated production program. Ozgun et al. (2004); Golldani and Moghaddam (2006) reported various plant heights for different chickpea varieties. Plant height significantly influenced by different supplementary treatments at different DAS (Figure 2). Supplemental irrigation along with aqueous $N$ before flowering produced the tallest plant height and control treatment produced the shortest plant. Supplementary spraying ensured favorable condition for chickpea plant with longest plant. Junttila (1992) reported that auxin type growth regulators had significant effect on the elongation of shoots of crop plants. Fallah et al. (2005) recorded longest plant growth applying supplementary irrigation.

The interaction effect of chickpea varieties and different supplementary treatments on plant height was significantly reflected at different stages of chickpea (Table 2). At 60, 75, 90 and 105 DAS the tallest plant $(30.13,32.66,37.13$ and 39.31 $\mathrm{cm}$, respectively) was recorded from BARI chola 9 with supplemental irrigation along with aqueous $\mathrm{N}$ before flowering, while the shortest plant (21.13, $24.13,25.00$ and $25.94 \mathrm{~cm}$, respectively) was obtained from BARI chola 8 with control treatment i.e., no spray at flowering and afterwards. Kang et al. (2008) reported that to achieve yield potential, crops should be irrigated over the whole of crop growth.

\section{Number of branches plant ${ }^{-1}$}

Chickpea varieties showed significant variation on number of branches plant ${ }^{-1}$ at different DAS (Figure 3). Management practices influence the number of branches plant ${ }^{-1}$ but varieties itself also manipulated it. Ozgun et al. (2004); Solaiman et al. (2007) reported that chickpea cultivars differed 

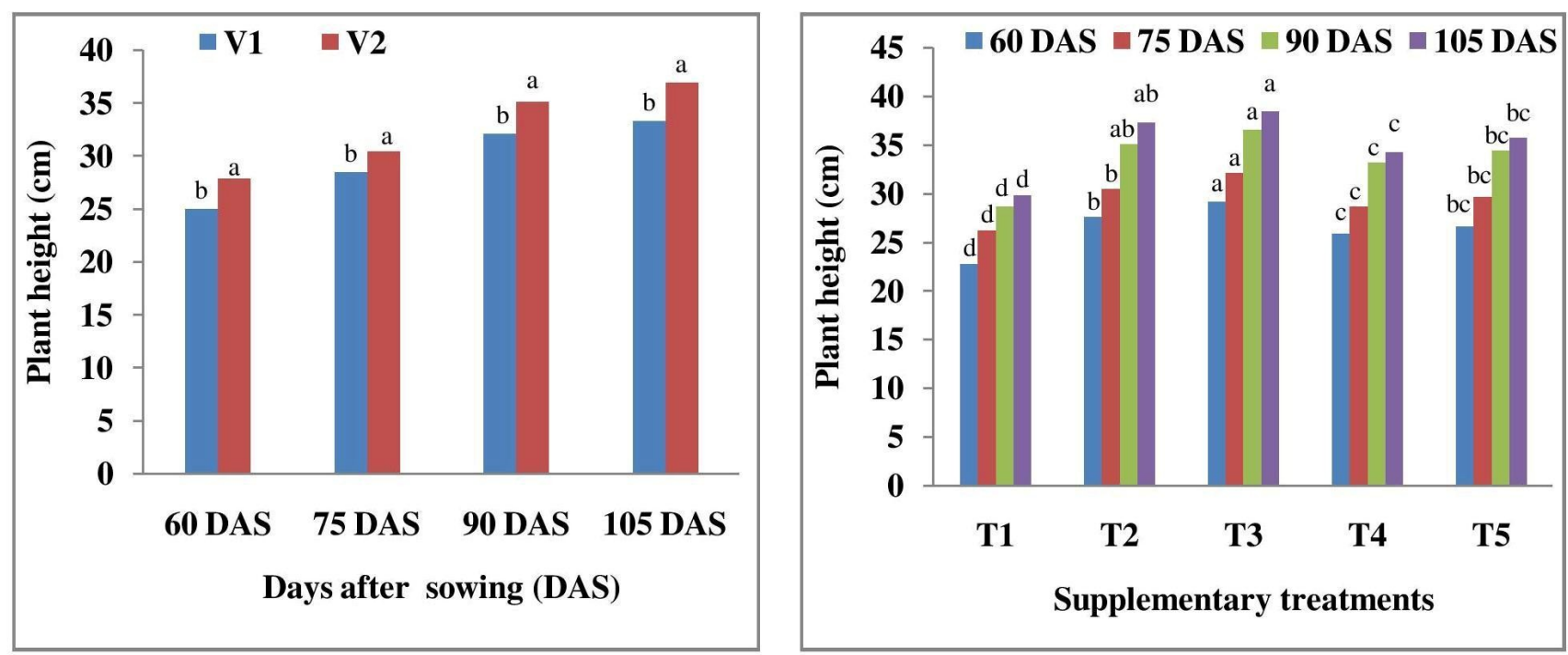

$\mathrm{V}_{1}$ : B ARI chola 8; $\mathrm{V}_{2}$ : BARI chola 9; $\mathrm{T}_{1}$ : Control i.e., no spray at flowering and afterwards; $\mathrm{T}_{2}$ : Supplemental irrigation before flowering; $\mathrm{T}_{3}$ : Supplemental irrigation + Aqueous $\mathrm{N}$ before flowering; $\mathrm{T}_{4}$ : $\mathrm{PRH}$ (a phytohormone) spray before flowering; $\mathrm{T}_{5}:$ Kinetine spray before flowering

Figure 1. Effect of variety on plant height of chickpea at different DAS (LSD value $=1.959,1.630,2.958$ and 3.520 at 60, 75, 90 and 105 DAS, respectively)
Figure 2. Effect of supplementary treatments on plant height of chickpea at different DAS (LSD value $=0.975$, $1.130,1.665$ and 1.600 at $60,75,90$ and 105 DAS, respectively)

Table 2. Interaction effect of variety and supplementary nitrogen, irrigation and hormone spray on plant height of chickpea at different DAS

\begin{tabular}{|c|c|c|c|c|}
\hline \multirow{2}{*}{ Treatment combination } & \multicolumn{4}{|c|}{ Plant height $(\mathrm{cm})$ at } \\
\hline & 60 DAS & 75 DAS & 90 DAS & 105 DAS \\
\hline $\mathrm{V}_{1} \mathrm{~T}_{1}$ & $21.13 \mathrm{~g}$ & $24.13 \mathrm{f}$ & $25.00 \mathrm{e}$ & $25.94 \mathrm{e}$ \\
\hline $\mathrm{V}_{1} \mathrm{~T}_{2}$ & $26.62 \mathrm{~cd}$ & $30.23 \mathrm{bc}$ & $34.74 \mathrm{a}-\mathrm{c}$ & $36.65 \mathrm{~b}$ \\
\hline $\mathrm{V}_{1} \mathrm{~T}_{3}$ & $28.16 \mathrm{~b}$ & $31.62 \mathrm{ab}$ & $36.00 \mathrm{a}$ & $37.47 \mathrm{ab}$ \\
\hline $\mathrm{V}_{1} \mathrm{~T}_{4}$ & $23.43 \mathrm{f}$ & $26.94 \mathrm{e}$ & $31.56 \mathrm{~d}$ & $32.45 \mathrm{~d}$ \\
\hline $\mathrm{V}_{1} \mathrm{~T}_{5}$ & 25.46 de & $29.25 \mathrm{~cd}$ & $33.07 \mathrm{~b}-\mathrm{d}$ & $33.99 \mathrm{~cd}$ \\
\hline $\mathrm{V}_{2} \mathrm{~T}_{1}$ & 24.44 ef & $28.33 \mathrm{de}$ & $32.33 \mathrm{~cd}$ & $33.74 \mathrm{~cd}$ \\
\hline $\mathrm{V}_{2} \mathrm{~T}_{2}$ & $28.51 \mathrm{~b}$ & $30.74 \mathrm{bc}$ & $35.36 \mathrm{ab}$ & $37.89 \mathrm{ab}$ \\
\hline $\mathrm{V}_{2} \mathrm{~T}_{3}$ & $30.13 \mathrm{a}$ & $32.66 \mathrm{a}$ & $37.13 \mathrm{a}$ & $39.31 \mathrm{a}$ \\
\hline $\mathrm{V}_{2} \mathrm{~T}_{4}$ & $28.24 \mathrm{~b}$ & $30.39 \mathrm{bc}$ & $34.74 \mathrm{a}-\mathrm{c}$ & $36.08 \mathrm{bc}$ \\
\hline $\mathrm{V}_{2} \mathrm{~T}_{5}$ & $27.79 \mathrm{bc}$ & $29.99 \mathrm{~b}-\mathrm{d}$ & $35.75 \mathrm{a}$ & $37.37 \mathrm{ab}$ \\
\hline $\operatorname{LSD}_{(0.05)}$ & 1.38 & 1.60 & 2.36 & 2.26 \\
\hline Level of significance & * & $* *$ & $* *$ & $* *$ \\
\hline CV (\%) & 3.01 & 3.14 & 4.05 & 3.72 \\
\hline
\end{tabular}

**: significant at $\mathrm{p} \leq 0.01, *$ : significant at $\mathrm{p} \leq 0.05$

Values followed by the same letters do not differ at $5 \%$ level of significance

$\mathrm{V}_{1}$ : BARI chola 8; $\mathrm{V}_{2}$ : BARI chola 9; $\mathrm{T}_{1}$ : Control i.e., no spray at flowering and afterwards; $\mathrm{T}_{2}$ : Supplemental irrigation before flowering; $\mathrm{T}_{3}$ : Supplemental irrigation + Aqueous $\mathrm{N}$ before flowering; $\mathrm{T}_{4}$ : $\mathrm{PRH}$ (a phytohormone) spray before flowering; $T_{5}$ : Kinetine spray before flowering

significantly with respect to number of branches plant $^{-1}$. Supplemental nitrogen, irrigation and hormone showed significant variation on number of branches plant ${ }^{-1}$ at $60,75,90$ and 105 DAS
(Figure 4). Supplemental irrigation along with aqueous $\mathrm{N}$ before flowering ensured favourable growth condition which produced maximum branches plant ${ }^{-1}$ of chickpea plant. Hafiz (2000) 

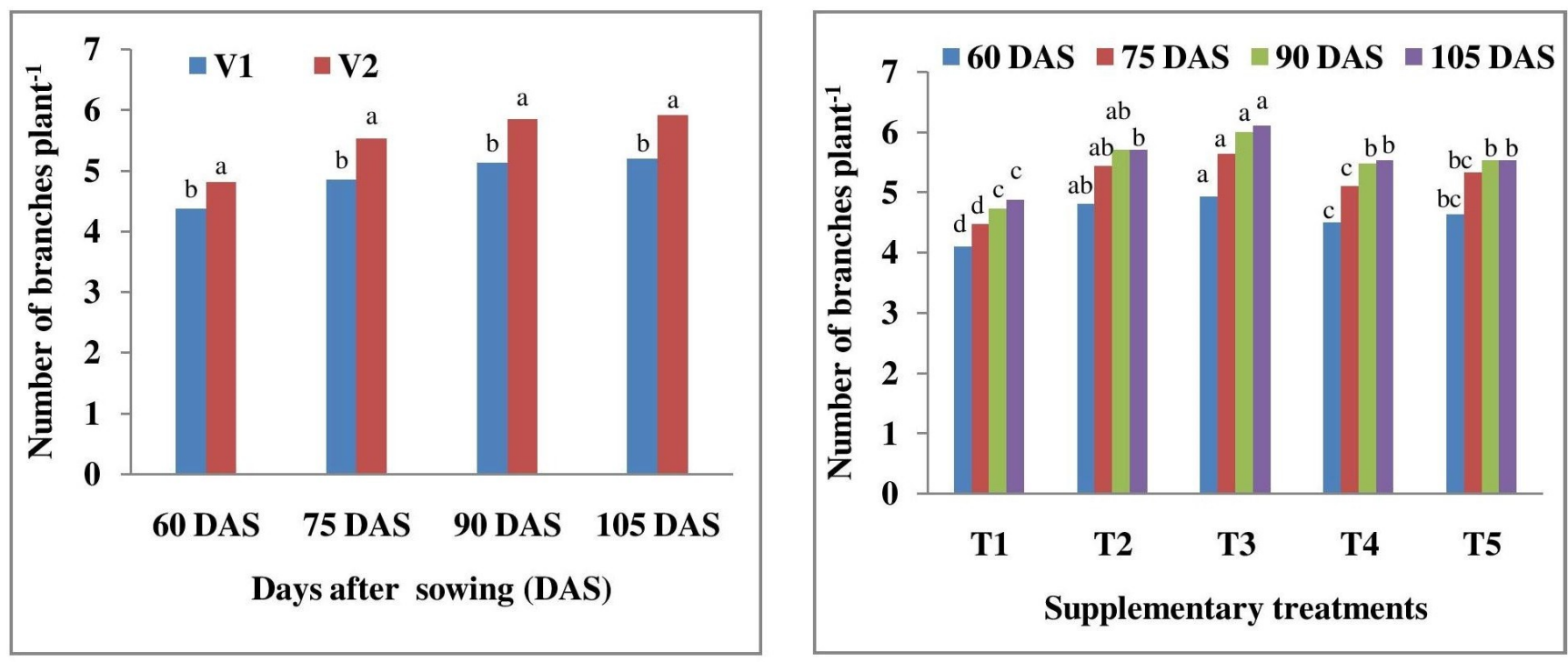

$\mathrm{V}_{1}$ : BARI chola 8; $\mathrm{V}_{2}$ : BARI chola 9; $\mathrm{T}_{1}$ : Control i.e., no spray at flowering and afterwards; $\mathrm{T}_{2}$ : Supplemental irrigation before flowering; $\mathrm{T}_{3}$ : Supplemental irrigation + Aqueous $\mathrm{N}$ before flowering; $\mathrm{T}_{4}$ : PRH (a phytohormone) spray before flowering; $\mathrm{T}_{5}$ : Kinetine spray before flowering

Figure 3. Effect of variety on number of branches plant ${ }^{-1}$ of chickpea at different DAS $($ LSD value $=0.172,0.455,0.489$ and 0.344 at $60,75,90$ and 105 DAS, respectively)
Figure 4. Effect of supplementary treatments on number of branches plant $^{-1}$ of chickpea at different DAS (LSD value = $0.242,0.274,0.391$ and 0.373 at $60,75,90$ and 105 DAS, respectively)

Table 3. Interaction effect of variety and supplementary nitrogen, irrigation and hormone spray on number of branches plant ${ }^{-1}$ of chickpea at different DAS

\begin{tabular}{|c|c|c|c|c|}
\hline \multirow{2}{*}{ Treatment combination } & \multicolumn{4}{|c|}{ Number of branches plant ${ }^{-1}$ at } \\
\hline & 60 DAS & 75 DAS & 90 DAS & 105 DAS \\
\hline $\mathrm{V}_{1} \mathrm{~T}_{1}$ & $3.60 \mathrm{e}$ & $3.80 \mathrm{~d}$ & $4.07 \mathrm{~d}$ & $4.20 \mathrm{e}$ \\
\hline $\mathrm{V}_{1} \mathrm{~T}_{2}$ & $4.67 \mathrm{a}-\mathrm{c}$ & $5.07 \mathrm{bc}$ & $5.40 \mathrm{bc}$ & $5.40 \mathrm{~cd}$ \\
\hline $\mathrm{V}_{1} \mathrm{~T}_{3}$ & $4.87 \mathrm{a}-\mathrm{c}$ & $5.33 \mathrm{~b}$ & $5.53 \mathrm{bc}$ & $5.67 \mathrm{~b}-\mathrm{d}$ \\
\hline $\mathrm{V}_{1} \mathrm{~T}_{4}$ & $4.20 \mathrm{~d}$ & $4.80 \mathrm{c}$ & $5.13 \mathrm{c}$ & $5.13 \mathrm{~d}$ \\
\hline $\mathrm{V}_{1} \mathrm{~T}_{5}$ & $4.53 \mathrm{~cd}$ & $5.27 \mathrm{~b}$ & $5.53 \mathrm{bc}$ & $5.53 \mathrm{~b}-\mathrm{d}$ \\
\hline $\mathrm{V}_{2} \mathrm{~T}_{1}$ & $4.60 \mathrm{bc}$ & $5.13 \mathrm{bc}$ & $5.40 \mathrm{bc}$ & $5.53 \mathrm{~b}-\mathrm{d}$ \\
\hline $\mathrm{V}_{2} \mathrm{~T}_{2}$ & $4.93 \mathrm{ab}$ & $5.80 \mathrm{a}$ & $6.00 \mathrm{ab}$ & $6.00 \mathrm{~b}$ \\
\hline $\mathrm{V}_{2} \mathrm{~T}_{3}$ & $5.00 \mathrm{a}$ & $5.93 \mathrm{a}$ & $6.47 \mathrm{a}$ & $6.53 \mathrm{a}$ \\
\hline $\mathrm{V}_{2} \mathrm{~T}_{4}$ & $4.80 \mathrm{a}-\mathrm{c}$ & $5.40 \mathrm{~b}$ & $5.80 \mathrm{~b}$ & $5.93 \mathrm{bc}$ \\
\hline $\mathrm{V}_{2} \mathrm{~T}_{5}$ & $4.73 \mathrm{a}-\mathrm{c}$ & $5.40 \mathrm{~b}$ & $5.53 \mathrm{bc}$ & $5.53 \mathrm{~b}-\mathrm{d}$ \\
\hline $\operatorname{LSD}_{(0.05)}$ & 0.34 & 0.39 & 0.55 & 0.53 \\
\hline Level of significance & $* *$ & $* *$ & $*$ & $*$ \\
\hline CV (\%) & 4.30 & 4.29 & 5.82 & 5.50 \\
\hline
\end{tabular}

**: significant at $\mathrm{p} \leq 0.01,{ }^{*}$ : significant at $\mathrm{p} \leq 0.05$

Values followed by the same letters do not differ at $5 \%$ level of significance

$\mathrm{V}_{1}$ : BARI chola 8; $\mathrm{V}_{2}$ : BARI chola 9; $\mathrm{T}_{1}$ : Control i.e., no spray at flowering and afterwards; $\mathrm{T}_{2}$ : Supplemental irrigation before flowering; $\mathrm{T}_{3}$ : Supplemental irrigation + Aqueous $\mathrm{N}$ before flowering; $\mathrm{T}_{4}$ : PRH (a phytohormone) spray before flowering; $\mathrm{T}_{5}$ : Kinetine spray before flowering

reported that late supplementary foliar spraying with aqueous solution of $1 \%$ urea significantly increased all the studied growth characters. Eid et al. (1991) and Iqbal et al. (2001) reported that branches of pea plants increased with foliar application of growth regulators.
Chickpea varieties and different supplementary treatments showed significant differences on number of branches plant ${ }^{-1}$ at different DAS (Table 3). At $60,75,90$ and 105 DAS the maximum number of branches plant ${ }^{-1}(5.00,5.93,6.47$ and 6.53, respectively) was attained from BARI chola 9 

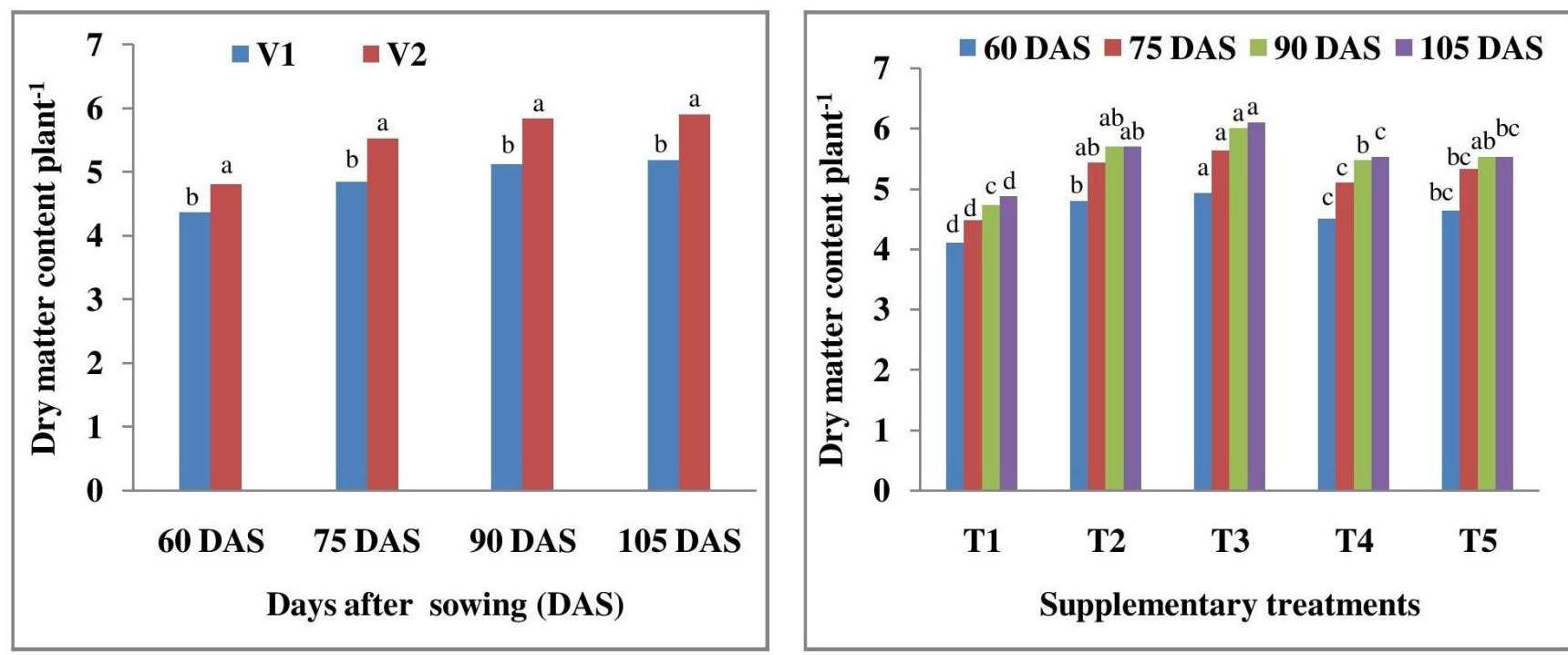

$\mathrm{V}_{1}$ : BARI chola 8; $\mathrm{V}_{2}$ : BARI chola 9; $\mathrm{T}_{1}$ : Control i.e., no spray at flowering and afterwards; $\mathrm{T}_{2}$ : Supplemental irrigation before flowering; $\mathrm{T}_{3}$ : Supplemental irrigation + Aqueous $\mathrm{N}$ before flowering; $\mathrm{T}_{4}$ : $\mathrm{PRH}$ (a phytohormone) spray before flowering; $\mathrm{T}_{5}$ : Kinetine spray before flowering

Figure 5. Effect of variety on dry matter content plant ${ }^{-1}$ of chickpea at different DAS (LSD value $=0.436,0.322,0.497$ and 0.272 at $60,75,90$ and 105 DAS)
Figure 6. Effect of supplementary treatments on dry matter content plant $^{-1}$ of chickpea at different DAS (LSD value $=0.242,0.274,0.391$ and 0.373 at $60,75,90$ and 105 DAS)

Table 4. Effect of variety and supplementary nitrogen, irrigation and hormone spray on dry matter content plant $^{-1}$ of chickpea at different DAS

\begin{tabular}{|c|c|c|c|c|}
\hline \multirow{2}{*}{ Treatment combination } & \multicolumn{4}{|c|}{ Dry matter content plant $^{-1}(\mathrm{~g})$ at } \\
\hline & 60 DAS & 75 DAS & 90 DAS & 105 DAS \\
\hline $\mathrm{V}_{1} \mathrm{~T}_{1}$ & $3.14 \mathrm{~d}$ & $3.83 \mathrm{f}$ & $4.10 \mathrm{e}$ & $4.25 \mathrm{e}$ \\
\hline $\mathrm{V}_{1} \mathrm{~T}_{2}$ & $4.15 \mathrm{~b}$ & $5.11 \mathrm{~b}-\mathrm{d}$ & $5.68 \mathrm{bc}$ & $6.08 \mathrm{~b}$ \\
\hline $\mathrm{V}_{1} \mathrm{~T}_{3}$ & $4.27 \mathrm{~b}$ & $4.80 \mathrm{c}-\mathrm{e}$ & $5.23 \mathrm{~cd}$ & $5.47 \mathrm{~cd}$ \\
\hline $\mathrm{V}_{1} \mathrm{~T}_{4}$ & $3.65 \mathrm{c}$ & $4.57 \mathrm{de}$ & $5.33 \mathrm{bc}$ & $5.67 \mathrm{bc}$ \\
\hline $\mathrm{V}_{1} \mathrm{~T}_{5}$ & $3.97 \mathrm{bc}$ & $4.73 \mathrm{de}$ & $5.35 \mathrm{bc}$ & $5.68 \mathrm{bc}$ \\
\hline $\mathrm{V}_{2} \mathrm{~T}_{1}$ & $3.55 \mathrm{~cd}$ & $4.40 \mathrm{e}$ & $4.66 \mathrm{de}$ & $5.05 \mathrm{~d}$ \\
\hline $\mathrm{V}_{2} \mathrm{~T}_{2}$ & $4.44 \mathrm{~b}$ & $5.57 \mathrm{~b}$ & $5.98 \mathrm{~b}$ & $6.21 \mathrm{~b}$ \\
\hline $\mathrm{V}_{2} \mathrm{~T}_{3}$ & $5.23 \mathrm{a}$ & $6.25 \mathrm{a}$ & $6.75 \mathrm{a}$ & $7.02 \mathrm{a}$ \\
\hline $\mathrm{V}_{2} \mathrm{~T}_{4}$ & $4.24 \mathrm{~b}$ & $5.07 \mathrm{~b}-\mathrm{d}$ & $5.68 \mathrm{bc}$ & $5.86 \mathrm{bc}$ \\
\hline $\mathrm{V}_{2} \mathrm{~T}_{5}$ & $4.39 \mathrm{~b}$ & $5.37 \mathrm{bc}$ & $5.82 \mathrm{bc}$ & $6.04 \mathrm{~b}$ \\
\hline $\operatorname{LSD}_{(0.05)}$ & 0.47 & 0.55 & 0.62 & 0.49 \\
\hline Level of significance & $*$ & $*$ & $*$ & $* *$ \\
\hline CV (\%) & 6.63 & 6.40 & 6.60 & 4.94 \\
\hline
\end{tabular}

**: significant at $\mathrm{p} \leq 0.01, *$ : significant at $\mathrm{p} \leq 0.05$

Values followed by the same letters do not differ at $5 \%$ level of significance

$\mathrm{V}_{1}$ : BARI chola 8; $\mathrm{V}_{2}$ : BARI chola 9; $\mathrm{T}_{1}$ : Control i.e., no spray at flowering and afterwards; $\mathrm{T}_{2}$ : Supplemental irrigation before flowering; $\mathrm{T}_{3}$ : Supplemental irrigation + Aqueous $\mathrm{N}$ before flowering; $\mathrm{T}_{4}$ : $\mathrm{PRH}$ (a phytohormone) spray before flowering; $T_{5}$ : Kinetine spray before flowering

and supplemental irrigation along with aqueous $\mathrm{N}$ before flowering, whereas the minimum number of branches plant ${ }^{-1}(3.60,3.80,4.07$ and 4.20 , respectively) from BARI chola 8 with control treatment i.e., no spray at flowering and afterwards.

\section{Dry matter content plant ${ }^{-1}$}

Dry matter content plant ${ }^{-1}$ varied significantly between varieties at different DAS (Figure 5). Data revealed that at $60,75,90$ and 105 DAS the maximum dry matter content plant ${ }^{-1}$ was found from BARI chola 9, while the minimum was 
Table 5. Effect of variety and/or supplemental nitrogen, irrigation and hormone spray on flower, pod, total dropping, pod remaining, pod length and seed yield of chickpea

\begin{tabular}{|c|c|c|c|c|c|c|}
\hline Treatments & $\begin{array}{c}\text { Flower } \\
\text { dropping (\%) }\end{array}$ & $\begin{array}{c}\text { Pod dropping } \\
(\%)\end{array}$ & $\begin{array}{c}\text { Total } \\
\text { dropping } \\
(\%)\end{array}$ & $\begin{array}{c}\text { Pod remaining } \\
(\%)\end{array}$ & $\begin{array}{l}\text { Pod length } \\
\text { (cm) }\end{array}$ & $\begin{array}{l}\text { Seed yield } \\
\quad\left(\mathrm{t} \mathrm{ha}^{-1}\right)\end{array}$ \\
\hline \multicolumn{7}{|l|}{ Variety } \\
\hline $\mathrm{V}_{1}$ & $64.98 \mathrm{a}$ & $3.79 \mathrm{a}$ & $68.77 \mathrm{a}$ & $31.23 \mathrm{~b}$ & $1.75 \mathrm{~b}$ & $1.43 \mathrm{~b}$ \\
\hline $\mathrm{V}_{2}$ & $60.27 \mathrm{~b}$ & $3.62 \mathrm{~b}$ & $63.89 \mathrm{a}$ & $36.11 \mathrm{a}$ & $1.85 \mathrm{a}$ & $1.69 \mathrm{a}$ \\
\hline $\operatorname{LSD}_{(0.05)}$ & 4.42 & 0.15 & 4.45 & 4.45 & 0.09 & 0.12 \\
\hline Level of significance & $*$ & $*$ & $*$ & $*$ & $*$ & * \\
\hline CV (\%) & 4.49 & 2.56 & 5.27 & 8.41 & 3.04 & 4.97 \\
\hline \multicolumn{7}{|c|}{ Supplemental treatments } \\
\hline $\mathrm{T}_{1}$ & $67.07 \mathrm{a}$ & $4.82 \mathrm{a}$ & $71.88 \mathrm{a}$ & $28.12 \mathrm{c}$ & $1.63 \mathrm{~d}$ & $1.25 \mathrm{c}$ \\
\hline $\mathrm{T}_{2}$ & $61.57 \mathrm{bc}$ & $3.27 \mathrm{c}$ & $64.83 \mathrm{bc}$ & $35.17 \mathrm{ab}$ & $1.89 \mathrm{~b}$ & $1.71 \mathrm{a}$ \\
\hline $\mathrm{T}_{3}$ & $59.17 \mathrm{c}$ & $3.10 \mathrm{~d}$ & $62.27 \mathrm{c}$ & $37.73 \mathrm{a}$ & $1.95 \mathrm{a}$ & $1.84 \mathrm{a}$ \\
\hline $\mathrm{T}_{4}$ & $62.70 \mathrm{~b}$ & $3.62 \mathrm{~b}$ & $66.32 \mathrm{~b}$ & $33.68 \mathrm{~b}$ & $1.74 \mathrm{c}$ & $1.49 \mathrm{~b}$ \\
\hline $\mathrm{T}_{5}$ & $62.63 \mathrm{~b}$ & $3.72 \mathrm{~b}$ & $66.35 \mathrm{~b}$ & $33.65 \mathrm{~b}$ & $1.79 \mathrm{c}$ & $1.51 \mathrm{~b}$ \\
\hline $\operatorname{LSD}_{(0.05)}$ & 2.66 & 0.10 & 2.66 & 2.66 & 0.06 & 0.17 \\
\hline Level of significance & $* *$ & $* *$ & $* *$ & $* *$ & $* *$ & $* *$ \\
\hline $\mathrm{CV}(\%)$ & 3.48 & 2.26 & 3.27 & 6.45 & 2.49 & 9.73 \\
\hline \multicolumn{7}{|l|}{ Treatment combination } \\
\hline $\mathrm{V}_{1} \mathrm{~T}_{1}$ & $71.20 \mathrm{a}$ & $4.97 \mathrm{a}$ & $76.17 \mathrm{a}$ & $23.83 \mathrm{e}$ & $1.45 \mathrm{e}$ & $1.19 \mathrm{e}$ \\
\hline $\mathrm{V}_{1} \mathrm{~T}_{2}$ & $63.40 \mathrm{~b}-\mathrm{d}$ & $3.30 \mathrm{f}$ & $66.70 \mathrm{~b}-\mathrm{d}$ & $33.30 \mathrm{~b}-\mathrm{d}$ & $1.90 \mathrm{ab}$ & $1.55 \mathrm{~cd}$ \\
\hline $\mathrm{V}_{1} \mathrm{~T}_{3}$ & $61.07 \mathrm{~b}-\mathrm{e}$ & $3.13 \mathrm{gh}$ & 64.20 c-e & $35.80 \mathrm{a}-\mathrm{c}$ & $1.93 \mathrm{a}$ & $1.74 \mathrm{a}-\mathrm{c}$ \\
\hline $\mathrm{V}_{1} \mathrm{~T}_{4}$ & $64.33 \mathrm{bc}$ & $3.73 \mathrm{~cd}$ & $68.07 \mathrm{bc}$ & $31.93 \mathrm{~cd}$ & $1.70 \mathrm{~d}$ & $1.32 \mathrm{de}$ \\
\hline $\mathrm{V}_{1} \mathrm{~T}_{5}$ & $64.90 \mathrm{~b}$ & $3.80 \mathrm{c}$ & $68.70 \mathrm{~b}$ & $31.30 \mathrm{~d}$ & $1.75 \mathrm{~cd}$ & $1.33 \mathrm{de}$ \\
\hline $\mathrm{V}_{2} \mathrm{~T}_{1}$ & $62.93 \mathrm{~b}-\mathrm{d}$ & $4.67 \mathrm{~b}$ & $67.60 \mathrm{bc}$ & $32.40 \mathrm{~cd}$ & $1.80 \mathrm{c}$ & $1.32 \mathrm{de}$ \\
\hline $\mathrm{V}_{2} \mathrm{~T}_{2}$ & 59.73 de & $3.23 \mathrm{fg}$ & $62.97 \mathrm{de}$ & $37.03 \mathrm{ab}$ & $1.88 \mathrm{ab}$ & $1.87 \mathrm{ab}$ \\
\hline $\mathrm{V}_{2} \mathrm{~T}_{3}$ & $57.27 \mathrm{e}$ & $3.07 \mathrm{~h}$ & $60.33 \mathrm{e}$ & $39.67 \mathrm{a}$ & $1.96 \mathrm{a}$ & $1.94 \mathrm{a}$ \\
\hline $\mathrm{V}_{2} \mathrm{~T}_{4}$ & $61.07 \mathrm{~b}-\mathrm{e}$ & $3.50 \mathrm{e}$ & $64.57 \mathrm{~b}-\mathrm{d}$ & $35.43 \mathrm{~b}-\mathrm{d}$ & $1.79 \mathrm{c}$ & $1.65 \mathrm{bc}$ \\
\hline $\mathrm{V}_{2} \mathrm{~T}_{5}$ & 60.37 c-e & $3.63 \mathrm{de}$ & 64.00 c-e & $36.00 \mathrm{a}-\mathrm{c}$ & $1.83 \mathrm{bc}$ & $1.69 \mathrm{a}-\mathrm{c}$ \\
\hline $\operatorname{LSD}_{(0.05)}$ & 3.767 & 0.149 & 3.76 & 3.758 & 0.077 & 0.263 \\
\hline Level of significance & $*$ & $*$ & $*$ & $*$ & $* *$ & $*$ \\
\hline CV (\%) & 3.48 & 2.26 & 3.27 & 6.45 & 2.49 & 9.73 \\
\hline
\end{tabular}

**: significant at $\mathrm{p} \leq 0.01, *$ : significant at $\mathrm{p} \leq 0.05$

Values followed by the same letters do not differ at $5 \%$ level of significance

$\mathrm{V}_{1}$ : BARI chola 8; $\mathrm{V}_{2}$ : BARI chola 9; $\mathrm{T}_{1}$ : Control i.e., no spray at flowering and afterwards; $\mathrm{T}_{2}$ : Supplemental irrigation before flowering; $\mathrm{T}_{3}$ : Supplemental irrigation + Aqueous $\mathrm{N}$ before flowering; $\mathrm{T}_{4}: \mathrm{PRH}$ (a phytohormone) spray before flowering; $\mathrm{T}_{5}$ : Kinetine spray before flowering

recorded from BARI chola 8. Variability in chickpea varieties for dry matter content had been reported by Solaiman et al. (2007); Sadeghipour and Aghaei (2012). Statistically significant variation was recorded for dry matter content plant $^{-1}$ at different DAS due to supplemental nitrogen, irrigation and hormones (Figure 6). Supplemental irrigation along with aqueous $\mathrm{N}$ before flowering produced the highest dry matter content plant ${ }^{-1}$ at different DAS. Supplementary spraying ensured favourable condition for the growth of chickpea plant with optimum vegetative growth and the ultimate results was the highest dry matter content plant ${ }^{-1}$.

Dry matter content plant ${ }^{-1}$ at 60, 75, 90 and 105 DAS varied significantly due to the interaction 
effect of chickpea varieties and different supplementary treatments (Table 4). At 60, 75, 90 and 105 DAS the maximum dry matter content plant $^{-1}(5.23 \mathrm{~g}, \quad 6.25 \mathrm{~g}, \quad 6.57 \mathrm{~g}$ and $7.02 \mathrm{~g}$, respectively) was attained from BARI chola 9 and supplemental irrigation along with aqueous $\mathrm{N}$ before flowering and the minimum dry matter content plant ${ }^{-1}\left(3.14 \mathrm{~g}, 3.83 \mathrm{~g}, 4.10 \mathrm{~g}^{2}\right.$ and $4.25 \mathrm{~g}$, respectively) was recorded from BARI chola 8 with control treatment i.e., no spray at flowering and afterwards.

\section{Flower dropping}

The results regarding flower dropping of chickpea varieties are given in Table 4, which showed that flower dropping of chickpea varieties differed significantly (Table 5). The lower flower dropping $(60.27 \%)$ was recorded from BARI chola 9, whereas the higher flower dropping (64.98\%) was recorded from BARI chola 8. Flower dropping of chickpea showed statistically significant differences for different supplementary treatments that applied as supplementary nitrogen, irrigation and hormones (Table 5). The lowest flower dropping (59.17\%) was found from supplemental irrigation along with aqueous $\mathrm{N}$ before flowering, which was statistically similar (61.17\%) with supplemental irrigation before flowering and closely followed $(62.63 \%$ and $62.70 \%$, respectively) by kinetine spray before flowering and PRH spray before flowering, while the highest flower dropping (67.07\%) was observed from control treatment. This might be due to suppression of apical dominance and increase in lateral branching that results in more number of branches plant ${ }^{-1}$ which ultimately produced more number of flowers plant $^{-1}$. Increased number of flower production may also be attributed to more flower retention (Singh et al., 2014). Interaction effect of chickpea varieties and different supplementary treatments varied significantly in terms of flower dropping (Table 5). The lowest flower dropping (57.27\%) was recorded from BARI chola 9 and supplemental irrigation along with aqueous $\mathrm{N}$ before flowering and the highest flower dropping (71.20\%) from BARI chola 8 with control treatment i.e., no spray at flowering and afterwards. Singh (2002) reported that application of growth regulator had a significant effect on number of flowers plant ${ }^{-1}$ over water spray treatment.

\section{Pod dropping}

The statistical analysis for pod dropping of chickpea varieties showed significant variation (Table 5). The lower pod dropping (3.62\%) was observed from BARI chola 9, while the higher pod dropping (3.79\%) was found from BARI chola 8. Statistically significant variation was recorded for pod dropping of chickpea due to the application of different supplementary treatments that applied as supplementary nitrogen, irrigation and hormones (Table 5). The lowest pod dropping (3.10\%) was recorded from supplemental irrigation along with aqueous $\mathrm{N}$ before flowering, which was closely followed (3.27\%) by supplemental irrigation before flowering, while the highest pod dropping (4.82\%) was found from control i.e., no spray at flowering and afterwards which was closely followed $(3.72 \%$ and $3.62 \%$, respectively) by kinetine spray before flowering and PRH spray before flowering. Singh et al. (2014) reported that different growth regulation practices were significantly better than control in producing more number of pods. Interaction effect of chickpea varieties and different supplementary treatments showed significant differences on pod dropping (Table 5). The minimum pod dropping (3.07\%) was recorded from BARI chola 9 and supplemental irrigation along with aqueous $\mathrm{N}$ before flowering, whereas the maximum pod dropping (4.97\%) from BARI chola 8 with control treatment i.e., no spray at flowering and afterwards.

\section{Total dropping}

Significant difference was found in terms of total dropping in chickpea varieties (Table 5). The lower total dropping (63.89\%) was observed from BARI chola 9 and the higher (68.77\%) was found from BARI chola 8. Total dropping of chickpea showed significant variation for different supplementary treatments (Table 5). The lowest total dropping (62.27\%) was found from supplemental irrigation along with aqueous $\mathrm{N}$ before flowering, which was statistically similar (64.83\%) with supplemental irrigation before flowering and closely followed (66.32\% and $66.35 \%$, respectively) by PRH spray before flowering and kinetine spray before flowering and they were statistically similar, whereas the highest total dropping (71.88\%) was observed from control i.e., no spray at flowering and afterwards. Interaction effect of chickpea varieties and different supplementary treatments showed significant differences on total dropping (Table 5). The lowest total dropping (60.33\%) was observed from BARI chola 9 and supplemental irrigation along with aqueous $\mathrm{N}$ before flowering, while the highest total dropping (76.17\%) was found from BARI chola 8 with control treatment i.e., no spray at flowering and afterwards.

\section{Pod remaining}

Significant difference was recorded among the varieties with respect to the pod remaining (Table 5). The higher pod remaining (36.11\%) was found from BARI chola 9, while the lower pod remaining $(31.23 \%)$ from BARI chola 8. Different supplementary treatments that applied as supplementary nitrogen, irrigation and hormones showed significant variation in terms of pod remaining of chickpea (Table 5). The highest pod remaining $(37.73 \%)$ was found from supplemental 
irrigation along with aqueous $\mathrm{N}$ before flowering, which was statistically similar (35.17\%) with supplemental irrigation before flowering and closely followed (33.68\% and $33.65 \%$, respectively) by PRH spray before flowering and kinetine spray before flowering. The lowest pod remaining (28.12\%) was observed from control treatment i.e., no spray at flowering and afterwards. The increase in number of pods might be due to increase in number of branches and increase in total number of flowers plant ${ }^{-1}$ (Singh et al., 2014). Chickpea varieties and different supplementary treatments varied significantly for pod remaining due to interaction effect (Table 5). The highest pod remaining (39.67\%) was found from BARI chola 9 and supplemental irrigation along with aqueous $\mathrm{N}$ before flowering and the lowest (23.83\%) from BARI chola 8 with control treatment i.e., no spray at flowering and afterwards. Singh (2002) revealed that application of growth regulator had a significant bearing on number of pods plant ${ }^{-1}$ over water spray treatment.

\section{Pod length}

Pod length of chickpea varieties varied significantly under the present trial (Table 5). The longer pod $(1.85 \mathrm{~cm})$ was recorded from BARI chola 9, whereas the shorter pod $(1.75 \mathrm{~cm})$ was found from BARI chola 8. Different varieties responded differently for pod length to input supply, method of cultivation and the prevailing environment during the growing season. Significant variation was recorded in terms of pod length of chickpea for different supplementary treatments (Table 5). The longest pod $(1.95 \mathrm{~cm})$ was found from supplemental irrigation along with aqueous $\mathrm{N}$ before flowering, which was followed $(1.89 \mathrm{~cm})$ by supplemental irrigation before flowering. On the other hand, the shortest pod $(1.63 \mathrm{~cm})$ was recorded from control treatment which was followed $(1.74 \mathrm{~cm}$ and 1.79 $\mathrm{cm})$ by PRH spray before flowering and kinetine spray before flowering. Bicer et al. (2004) reported that pod length were higher under irrigated than rainfed conditions. Interaction effect of chickpea varieties and different supplementary treatments exerted significant differences on pod length (Table 5). The longest pod $(1.96 \mathrm{~cm})$ was found from BARI chola 9 and supplemental irrigation along with aqueous $\mathrm{N}$ before flowering, while the shortest pod $(1.45 \mathrm{~cm})$ was observed from BARI chola 8 with control treatment i.e., no spray at flowering and afterwards.

\section{Seed yield}

A significant effect of varieties was found on the seed yield of chickpea (Table 5). The higher seed yield (1.69 t ha-1) was observed from BARI chola 9, whereas the lower seed yield $\left(1.43 \mathrm{t} \mathrm{ha}^{-1}\right)$ was found from BARI chola 8. Varieties plays an important role in producing high yield of chickpea and yield also varied for different varieties might be due to genetical and environmental influences as well as management practices. Mukherjee and Singh (2005); Solaiman et al. (2007) reported that chickpea genotypes differed significantly with respect to seed yield. The results of this experiment showed that different supplementary treatments had significant effect on seed yield of chickpea (Table 5). The highest seed yield (1.84 t $\mathrm{ha}^{-1}$ ) was recorded from supplemental irrigation along with aqueous $\mathrm{N}$ before flowering, which was statistically similar (1.71 t ha-1) with supplemental irrigation before flowering and closely followed (1.51 t ha- $\mathrm{t}^{-1}$ and $1.49 \mathrm{t} \mathrm{ha}^{-1}$, respectively) by kinetine spray before flowering and PRH spray before flowering, while the lowest seed yield $\left(1.25 \mathrm{t} \mathrm{ha}^{-1}\right)$ was attained from control treatment. It was revealed that supplementary spraying of nitrogen, irrigation and hormones ensured favorable condition for the growth of mungbean plant with optimum vegetative growth and the ultimate results was the highest yield. Fallah et al. (2005) reported that planting Greet palong with supplementary irrigation, may lead to a significant increase in grain yield under dryland conditions. Palta et al. (2005) reported that the potential to increase yields of chickpea by application of foliar nitrogen near flowering in environments in which terminal droughts reduce yield. Hafiz (2000) reported that late supplementary foliar spraying with aqueous solution of 1\% urea significantly increased yield and yield quality compared to the unsprayed control. Mohammadi et al. (2006) reported that among phenological stages of chickpea, pod formation was the most sensitive to water deficit and that under water limitation conditions chickpea yield could be improved by irrigation at this stage. Seed yield of chickpea significantly varied due to interaction between varieties and different supplementary treatments (Table 5). The highest seed yield (1.94 $\left.\mathrm{t} \mathrm{ha}^{-1}\right)$ was found from BARI chola 9 with supplemental irrigation along with aqueous $\mathrm{N}$ before flowering and the lowest seed yield (1.19 t ha $\left.{ }^{-1}\right)$ from BARI chola 8 with control treatment i.e., no spray at flowering and afterwards. Increased seed yield might be cumulative result of increased number of branches, flowers and pods plant ${ }^{-1}$.

\section{Conclusion}

Different variety varied significantly for growth and reproductive behaviour of chickpea. Among the varieties, BARI chola 9 showed lower flower and pod droppings which resulted higher yield. Supplemental irrigation before flowering along with $5.5 \mathrm{~g}$ aqueous nitrogen $\mathrm{m}^{-2}$ showed significant role to reduce flower and pod droppings of chickpea. Considering the findings of the present study, it is concluded that BARI chola 9 cultivated with applying supplemental irrigation along with aqueous $\mathrm{N}$ before flowering gave better growth, lower droppings and higher yield of chickpea. 


\section{Competing interests}

The authors declares that they have no competing interests.

\section{Acknowledgements}

The authors acknowledge the funding of this study from the Ministry of Science and TechnologyGovernment of the People's Republic of Bangladesh.

\section{References}

Ayallew, D., and R. A. Tabbada. 1987. Influence of soil moisture levels on the growth and development of the mungbean plant (Vigna radiata L.). Nat Applied Sci Bulletin 39(4): 273-280.

Aziz, M. A., M. A. Khan, and S. Shah. 1960. Causes of low setting of seed in gram (Cicer arietinum). Agric Pakistan 11(1): 37-48.

BARC. 2005. Fertilizer Recommendation Guide. Bangladesh Agriculture Research Council, Farmgate, Dhaka, p. 219.

BBS (Bangladesh Bureau of Statistics). 2010. Yearbook of Agricultural Statistics of Bangladesh. Planning Division. Ministry of Planning. Government of the People's Republic of Bangladesh. Dhaka, pp. 54-111.

Bicer, B. T., A. N. Kalender, and D. Sakar. 2004. The effect of irrigation on spring-sown chickpea. J Agron 3(3): 154-158. doi:10.3923/ja.2004.154.158

Deolankar, K. P. 2005. Effect of fertigation on growth and yield of chickpea. J Maharashtra Agril Uni 30(2): 170-172.

Dharmender, K., K. D. Hujar, R Paliwal, and D. Kumar. 1996. Yield and yield attributes of chickpea as influenced by $\mathrm{GA}_{3}$ and NAA. Crop Res Hisar 12(1): 120-122.

Eid, S. M. M., H. H. Abbas, and F. A. Abu-Sedra. 1991. Effect of GA foliar spray on plant growth, chemical composition, flowering, pod yield and chemical composition of green seeds for pea plants grown under salinity stress. Ann Agril Sci, Moshtohor (Egypt) 30: 1443-1458.

Fallah, S., P. Ehsanzadeh, and M. Daneshvar. 2005. Grain yield and yield components in three chickpea genotypes under dryland conditions with and without supplementary irrigation at different plant densities in Khorram-Abad, Lorestan. Iranian J Agril Sci 36(3): 719-731.

FAO (Food and Agriculture Organization of the United Nations). 2010. Agricultural production year book/ or http://faostat.fao.org.

Freed, D. R. 1986. MSTAT-C programme. Crop and Soil Science Department. Michigan State University, USA.

Golldani, A., and P. R. Moghaddam. 2006. Effect of different irrigation levels on phenology, physiology characteristics, and yield components of three chickpea (Cicer arietinum L.) cultivars in Mashhad. Agril Sci Tech 20(3): 21-32.

Gomez, K. A., and A. A. Gomez. 1984. Statistical procedures for Agricultural Research. Jhon Wiley and Sons, New York.

Hafiz, S. I. 2000. Response of three chickpea cultivars to late foliar spraying with urea as a supplement for early soil applied nitrogen in sandy soils. Ann Agril Sci 38(1): 31-46.

Iqbal, H. F., A. Tahir, M. N. Khalid, I. Haq, and A. N. Ahmad. 2001. Response of chickpea growth towards foliar application of Gibberellic acid at different growth stages. Pakistan J Biol Sci 4(4): 433-434. doi:10.3923/pjbs.2001.433.434

Junttila, O. 1992. Gibberellin and elongation growth. Nordisk Jordbruksforskning 74: 74.

Kang, S., B. A. McKenzie and G. D. Hill. 2008. Effect of irrigation on growth and yield of Kabuli chickpea (Cicer arietinum L.) and narrowleafed lupin (Lupinus angustifolius L.). Agron New Zealand 38: 11-32.

Kumar, S., B. R. T Singh, and R. C. Tyagi. 1995. Effect of irrigation on growth parameters of lentil (Lens culinaris L.). In: Field Crop Abst. 48(5): 428-29.

Mansoor, M. 2007. Evaluation of various agronomic management practices for increased productivity of mungbean (Vigna radiata $\mathrm{L}$.). Ph. D. Thesis, Dept of Agron, Faculty of Agric, Gomal University, Dera Islam Khan.

Miah, M. G., O. Hirota, and J. Chikushi. 1991. Influence of water status, photosynthesis rate and plant growth under different temperatures and water regimes during pod formation phase of mungbean (Vigna radiata). J Faculty Agric Kyushu Uni 41(1): 1728.

Mohammadi, G., K. G. Golezani, A. Javanshir, and M. Moghaddam. 2006. The influence of water limitation on the yield of three chickpea cultivars. JWSS-Isfahan Uni Technol 10(2): 109-120.

Mukesh, K. 2006. Impact of the starter doses of nitrogen on nodulation, yield and yield attributes of chickpea under irrigated conditions. Int J Agril Sci 2(1): 253-255.

Mukherjee, D., and R. K. Singh. 2005. Influence of weed flora density on yield of chickpea cultivars. Indian J Pulses Res 18(2): 222.

Nayyar, H., S. Singh, S. Kaur, S. Kumar, and H. D. Upadhyaya. 2006. Differential sensitivity of macrocarpa and microcarpa types of chickpea (Cicer arietinum L.) to water stress: association of contrasting stress response with oxidative injury. J Integrative Plant Biol 48(11): $\quad 1318-1329 . \quad$ doi:10.1111/j.17447909.2006.00350.x

Ozgun, O. S., B. T. Bicer, and D. Sakar. 2004. Agronomic and morphological characters of chickpea under irrigated conditions in Turkey. Int J Agric Biol 6(4): 606-610.

Palta, J. A., A. S. Nandwal, K. Sunita, and N. C. Turner. 2005. Foliar nitrogen applications increase 
the seed yield and protein content in chickpea (Cicer arietinum L.) subject to terminal drought. Australian J Agril Res 56(2): 105-112. doi:10.1071/AR04118

Patel, R. G., M. P. Palel, H. C. Palel, and R. B. Palel. 1984. Effect of graded levels of nitrogen and phosphorus on growth, yield and economics of summer mungbean. Indian J Agron 29(3): 42-44.

Patil, A. A., S. M. Maniur, and U. G. Nalwadi. 1987. Effect $\mathrm{GA}_{3}$ and NAA on growth and yield of pulses. South Indian Hort 35(5): 393-394.

Sadeghipour, O. and P. Aghaei. 2012. Comparison of autumn and spring sowing on performance of chickpea (Cicer arietinum L.) varieties. Int J Biosci 2(6): 49-58.

Saraf, C. S., B. Baldev, M. Ali, and S. N. Slim. 1990. Improved cropping systems and alternative cropping practices, pp. 105-108. In: chickpea in the nineties: Proceedings of the Second International Workshop on Chickpea Improvement, ICRISAT Center, India Patencheru, A, pp. 502-524.

Singh, S. 2002. Studies on the modification of microclimate in chickpea (Cicer arietinum L.) through the ecological manipulations. M. Sc. Thesis, P. A. U., Ludhiana, India.

Singh, S., J. S. Deol, and A. S. Brar. 2014. Growth and yield of kabuli gram (Cicer arietinum L.) as influenced by plant growth regulation and sowing time. Crop Res 48(1, 2 \& 3): 32-37.

Solaiman, A. R. M., D. Hossain, and M. G. Rabbani. 2007. Influence of rhizobium inoculant and mineral nitrogen on some chickpea varieties. Bangladesh J Microbiol 24(2): 146-150. 